\title{
Clones de seringueira com resistência a ácaros
}

\author{
Helder Adriano de Souza da Silva (1); Marineide Rosa Vieira $\left(2^{*}\right)$; Walter Veriano Valério Filho ( ${ }^{2}$ ); \\ Maria de Souza Monteverde Cardoso (1); Janayna Correa Figueira ( $\left.{ }^{3}\right)$
}

(') Universidade Estadual Paulista "Julio de Mesquita Filho" (UNESP), Programa de Pós-Graduação em Agronomia/Sistemas de Produção, Ilha Solteira (SP).

(2) UNESP, Faculdade de Engenharia (FEIS), Ilha Solteira (SP).

(3) In memoriam.

(*) Autora correspondente: marineid@bio.feis.unesp.br

Recebido: 18/dez./2009; Aceito: 18/dez./2010.

\begin{abstract}
Resumo
A cultura da seringueira pode ser atacada pelos ácaros Calacarus heveae Feres (Eriophyidae) e Tenuipalpus heveae Baker (Tenuipalpidae). Dentre as opções para controle dessas pragas, o uso de material vegetal resistente pode ser uma boa alternativa. O objetivo deste trabalho foi identificar clones resistentes a esses ácaros, e assim, contribuir para o estabelecimento de estratégias de manejo dessas pragas na cultura. Os experimentos foram realizados no município de Votuporanga (SP), em área com plantas dispostas em um delineamento em blocos casualizados com quatro repetições. Foram coletadas folhas dos clones RRIM 600, PB 260, PB 330, PB 28/59, IAC 15, IAC 35, IAC 40, IAC 300, IAN 3156 e IRCA 111, mensalmente, para contagem dos ácaros em laboratório. A contagem foi realizada em oito folíolos por parcela, em duas áreas de $1 \mathrm{~cm}{ }^{2}$ por folíolo, na página superior para C. heveae e na página inferior para T. heveae. Os níveis de desfolhamento observados foram avaliados com uma escala de notas de 0 (ausência de desfolhamento) a 4 (acima de 75\%). 0 clone RRIM 600 foi utilizado como genótipo-padrão de suscetibilidade. Com base nos valores de número médio de ácaros por $\mathrm{cm}^{2}$ e notas de desfolhamento, os clones IAC 15, IAC 40, IAC 300, IAN 3156 e PB 28/59 foram considerados resistentes por não preferência e/ou antibiose a C. heveae e T. heveae. O clone IRCA 111 foi tolerante a C. heveae.
\end{abstract}

Palavras-chave: Hevea brasiliensis, Acari, Calacarus heveae, Tenuipalpus heveae.

\section{Rubber tree clones with resistance to mites}

\section{Abstract}

The rubber tree crop can be attacked by Calacarus heveae Feres (Eriophyidae) and Tenuipalpus heveae Baker (Tenuipalpidae) mites. Among the control options, the use of resistant genetic material can be a good alternative. The objective of this study was to identify clones showing resistance to these mites, and thus contribute to the establishment of management strategies of these pests. The experiments were carried out in the Votuporanga, State of São Paulo, in an area with plants arranged in a randomized block design with four replications. Leaves of the clones IAC 15, IAC 35, IAC 40, IAC 300, IAN 3156, IRCA 111, PB 260, PB 330, PB 28/59 and RRIM 600 were collected monthly for mite counting. The count was conducted in eight leaflets per plot, in two areas of $1 \mathrm{~cm}^{2}$ per leaflet, on the upper surface for $C$. heveae and lower surface for T. heveae. The defoliation levels observed were evaluated with a grade scale ranging from 0 (without defoliation) to 4 (above 75\%). RRIM 600 clone was used as standard susceptible genotype. Based on the values of average number of mites per $\mathrm{cm}^{2}$ and defoliation grades, the IAC 15, IAC 40, IAC 300, IAN 3156 and PB 28/59 clones were considered resistant by non preference and/or antibiosis to C. heveae and T. heveae. The IRCA 111 clone was tolerant to C. heveae.

Key words: Hevea brasiliensis, Acari, Calacarus heveae, Tenuipalpus heveae. 


\section{INTRODUÇÃO}

Áreas de cultivo de seringueira [Hevea brasiliensis (Willd. ex Adr. de Juss.) Müell. Arg.] podem ser atacadas por várias espécies de ácaros fitófagos, entre elas, o microácaro Calacarus heveae Feres (Eriophyidae) e o ácaro-plano-vermelho Tenuipalpus heveae Baker (Tenuipalpidae) (Feres et al., 2002; Ferla e Moraes, 2002; Hernandes e Feres, 2006a). Esses ácaros têm sido registrados na cultura, em elevados níveis populacionais, principalmente no período de fevereiro a junho (Vieira e Gomes, 1999; Hernandes e Feres, 2006b; Vis et al., 2006; Demite e Feres, 2007), provocando amarelecimento progressivo das folhas e posteriormente, o desfolhamento das plantas antes do período normal de senescência (VieIra, 2010).

No estabelecimento de estratégias de manejo que possam minimizar o efeito prejudicial dessas espécies, o uso de clones mais resistentes pode ser uma importante estratégia de controle e trazer muitos benefícios à heveicultura, por reduzir os custos de produção e as possibilidades de contaminação do ambiente devido à aplicação de acaricidas.

Novos clones de seringueira têm sido avaliados em programas de melhoramento genético no Brasil, com ênfase em produção, crescimento, vigor, crescimento do caule durante a sangria, espessura da casca virgem, boa regeneração de casca, tolerância à queda pelo vento, tolerância à seca do painel e resistência às principais doenças da região (GonçALVES e MARQues, 2008). A resistência a pragas, insetos e ácaros ainda é uma avaliação a ser incorporada aos programas de melhoramento.

A manifestação da resistência pode ser afetada por fatores da planta (idade); fatores da praga (raça, biótipo e tamanho da população) e fatores ambientais (climáticos e edáficos). Dessa forma, uma planta pode manifestar sua resistência em determinadas condiçóes e não manter esse caráter em condiçóes diferentes (Lara, 1991). Para que haja segurança nas informaçôes sobre resistência dos clones de seringueira aos ácaros-praga, é necessário que diversos experimentos sobre a interação entre clones e ácaros, em diferentes condiçôes, sejam realizados.

Materiais com resistência a ácaros fitófagos têm sido identificados por alguns autores. No Estado do Mato Grosso, município de Itiquira, foi observado que os clones GT1 e PB 217 foram mais resistentes ao desenvolvimento populacional das duas espécies de importância econômica, em comparaçáo com o clone RRIM 600, enquanto PR 255 foi semelhante quanto à infestação de $T$. heveae, porém mais resistente ao ataque de $C$. heveae (Daud e Feres, 2007). No mesmo local, Ferla e Moraes (2008) em levantamentos realizados nos clones PB 260 e PR 255, observaram bom desenvolvimento de $C$. heveae com amarelecimento dos folíolos nos dois materiais e queda prematura de fo- lhas no clone PB 260. Na busca de clones resistentes, a avaliação de progênies de seringueira também pode permitir a identificação de materiais promissores para futura clonagem (Vieira et al., 2009).

Assim, com base na necessidade de gerar informaçóes que possam auxiliar na recomendação de clones para plantio no Estado de Sáo Paulo, este trabalho teve por objetivo identificar clones resistentes aos ácaros $C$. heveae e $T$. heveae, contribuindo assim para o estabelecimento de estratégias de manejo dessas pragas na cultura.

\section{MATERIAL E MÉTODOS}

O experimento foi desenvolvido no município de Votuporanga, localizado em latitude $20^{\circ} 20^{\prime} \mathrm{S}$ e longitude $49^{\circ} 58^{\prime} \mathrm{W}$, com altitude de $510 \mathrm{~m}$. As avaliações foram realizadas durante dois ciclos produtivos - 2004/2005 e 2005/2006 - em área com plantas dispostas em um delineamento em blocos casualizados com quatro repetiçóes. Dez clones foram avaliados, RRIM 600, PB 260, PB 330, PB 28/59, IAC 15, IAC 35, IAC 40, IAC 300, IAN 3156 e IRCA 111. Cada parcela foi constituída de cinco linhas de seis plantas, considerando-se como área útil as doze plantas centrais.

Para a realização das amostragens foram marcadas duas plantas na área útil de cada parcela, sendo coletadas de cada uma delas duas extremidades de ramo, com aproximadamente $30 \mathrm{~cm}$ de comprimento, de pontos diferentes da copa. O material coletado foi acondicionado em sacos plásticos e levado ao laboratório para contagem dos ácaros. As coletas foram realizadas mensalmente de dezembro de 2004 a junho de 2005 e, posteriormente, de novembro de 2005 a junho de 2006.

Em laboratório, de cada parcela, foram retirados oito folíolos para contagem de $C$. heveae na página superior, e outros oito folíolos, para contagem de $T$. heveae na página inferior, sendo retirados dois de cada um dos quatro ramos coletados. Para $C$. heveae foi realizada avaliação em duas áreas de $1 \mathrm{~cm}^{2}$, em cada folíolo, uma de cada lado da nervura principal e, para $T$. heveae, em duas áreas de $1 \mathrm{~cm}^{2}$ por folíolo, uma sobre a nervura principal e outra sobre uma nervura lateral.

O nível de desfolhamento observado nas plantas foi avaliado com o uso de uma escala de notas de 0 a 4 , sugerida por Vieira e Gomes (1999), sendo 0 para ausência de desfolhamento; 1 - 0 a 25\% de desfolhamento; 2 - 25\% a $50 \%$; 3 - 50\% a $75 \%$ e 4 - acima de $75 \%$. Essas avaliaçôes foram realizadas mensalmente, de dezembro a agosto do ano seguinte.

Em cada período, os dados de número médio de ácaros por $\mathrm{cm}^{2}$ e notas de desfolhamento foram submetidos à análise de variância, e as médias agrupadas pelo teste de Scott-Knott, a 5\% de probabilidade. No primeiro caso, os dados foram transformados em $\log (x+2)$, por não haver 
aderência à normalidade (teste de Lilliefors), considerando-se para as notas, os dados originais. As análises foram realizadas com o Programa Sisvar (Ferreira, 2008)

As infestaçôes observadas em 2004/2005 foram comparadas às registradas em 2005/2006 com o uso do teste t, para cada espécie de ácaro, com o Programa SAEG (RIBEIRo Junior, 2001). O mesmo procedimento foi adotado para as notas de desfolhamento.

Os dados de temperatura, umidade relativa do ar e precipitação pluviométrica foram coletados no posto meteorológico existente no local. Os valores totais mensais de precipitação pluvial, registrados em cada período, de agosto a fevereiro do ano seguinte, foram comparados pelo teste t para amostras pareadas, com o programa SAEG (Ribeiro Junior, 2001).

Para a classificação dos clones quanto à resistência aos ácaros, o clone RRIM 600, o mais plantado no Estado de São Paulo, foi considerado o padrão de suscetibilidade. Assim, foram considerados suscetíveis os clones com número médio de ácaros semelhante ao registrado em RRIM 600 em pelo menos um dos períodos analisados. Também foi considerado suscetível o clone que apesar de ter menor número de ácaros recebeu nota de desfolhamento semelhante.

Clones com número médio de ácaros inferior ao registrado em RRIM 600, nos dois períodos avaliados, foram considerados resistentes por não-preferência e/ou antibiose. Quando, apesar de ter número médio de ácaros semelhante ao do clone-padrão, a nota de desfolhamento foi inferior, nos dois períodos, o clone foi considerado resistente por tolerância.

\section{RESULTADOS E DISCUSSÃO}

Houve diferença significativa entre os clones quanto aos números médios de ácaros, das duas espécies, registrados em cada período (Tabela 1).

Em 2004/2005, em seis clones houve níveis populacionais de $C$. heveae inferiores ao registrado em RRIM 600 e três foram semelhantes a ele $(\mathrm{F}=3,3 ; \mathrm{p}<0,01)$. Para $T$. heveae, em todos os clones, o desenvolvimento foi inferior ao do clone-padrão $(\mathrm{F}=7,20 ; \mathrm{p}<0,01)$. A nota média de desfolhamento foi semelhante à de RRIM 600 em três clones e inferior em seis $(F=6,32 ; p<0,01)$, ou seja, os clones IAC 15, IAC 40, IAC 300, IAN 3156, PB 28/59 e IRCA 111.

Para o período 2005/2006, apenas no clone IRCA 111 a população de $C$. heveae foi semelhante ao de RRIM 600, e em todos os outros o nível foi inferior $(F=7,27 ; p<0,01)$. Todos os clones tiveram menor desenvolvimento de $T$. heveae $(\mathrm{F}=5,01 ; \mathrm{p}<0,01)$. Em todos eles a nota de desfolhamento foi inferior à registrada para RRIM $600(\mathrm{~F}=6,74$; $\mathrm{p}<0,01)$. Os menores níveis de desfolhamento foram registrados nos clones IAC 15, IAC 40, PB 28/59 e IRCA 111.

Parte do desfolhamento observado na área experimental pode ter ocorrido em função de um processo natural de senescência. Entretanto, ao longo dos dois períodos, verificou-se grande quantidade de folíolos com sintomas das duas espécies de ácaros nas plantas e no chão da área, indicando ser essa a principal causa do desfolhamento.

$\mathrm{Na}$ classificação dos clones quanto a possíveis fatores de resistência, considerou-se que a resistência é relativa, o que implica comparação de duas ou mais plantas. Uma

Tabela 1. Número médio de ácaros $\left({ }^{1}\right)$ por $\mathrm{cm}^{2}$, das espécies $C$. heveae $(C h)$ e $T$. heveae $(T h)$, nota de desfolhamento $\left({ }^{2}\right)$, e tipo de resistência em clones de seringueira. Votuporanga (SP)

\begin{tabular}{|c|c|c|c|c|c|c|c|c|}
\hline \multirow{3}{*}{ Clones } & \multicolumn{3}{|c|}{ Período 2004/2005 } & \multicolumn{3}{|c|}{ Período 2005/2006 } & \multirow{2}{*}{\multicolumn{2}{|c|}{$\begin{array}{c}\text { Resistência } \\
\text { Tipo }\end{array}$}} \\
\hline & \multicolumn{2}{|c|}{ N. ${ }^{\circ}$ de indivíduos } & \multirow{2}{*}{ Nota $\left({ }^{3}\right)$} & \multicolumn{2}{|c|}{ No de indivíduos } & \multirow{2}{*}{ Nota $\left({ }^{3}\right)$} & & \\
\hline & Ch & Th & & Ch & Th & & Ch & Th \\
\hline IAC 15 & $0,71 \pm 0,19 b$ & $0,08 \pm 0,02 c$ & $1,22 \pm 0,08 \mathrm{~b}$ & $0,85 \pm 0,25 c$ & $0,29 \pm 0,06 b$ & $1,89 \pm 0,18 \mathrm{c}$ & $\mathrm{R}_{\mathrm{NP} / \mathrm{A}}$ & $\mathrm{R}_{\mathrm{NP} / \mathrm{A}}$ \\
\hline IAC 35 & $1,42 \pm 0,38 a$ & $0,09 \pm 0,02 c$ & $1,68 \pm 0,11$ a & $2,53 \pm 0,63 b$ & $0,05 \pm 0,01 c$ & $1,94 \pm 0,23 b$ & S & $\mathrm{S}$ \\
\hline IAC 40 & $0,86 \pm 0,26 b$ & $0,32 \pm 0,09 b$ & $1,36 \pm 0,08 \mathrm{~b}$ & $1,58 \pm 0,49 b$ & $0,17 \pm 0,03 c$ & $1,83 \pm 0,19 \mathrm{c}$ & $\mathrm{R}_{\mathrm{NP} / \mathrm{A}}$ & $\mathrm{R}_{\mathrm{NP} / \mathrm{A}}$ \\
\hline IAC 300 & $0,73 \pm 0,23 b$ & $0,07 \pm 0,03 c$ & $1,50 \pm 0,17 b$ & $1,64 \pm 0,37 b$ & $0,09 \pm 0,03 c$ & $1,99 \pm 0,22 b$ & $\mathrm{R}_{\mathrm{NP} / \mathrm{A}}$ & $\mathrm{R}_{\mathrm{NP} / \mathrm{A}}$ \\
\hline IAN 3156 & $0,58 \pm 0,22 b$ & $0,08 \pm 0,03 c$ & $1,33 \pm 0,08 b$ & $1,59 \pm 0,38 b$ & $0,05 \pm 0,02 c$ & $2,00 \pm 0,18 b$ & $\mathrm{R}_{\mathrm{NP} / \mathrm{A}}$ & $\mathrm{R}_{\mathrm{NP} / \mathrm{A}}$ \\
\hline PB 260 & $0,99 \pm 0,26 b$ & $0,18 \pm 0,06 c$ & $1,85 \pm 0,17$ a & $1,68 \pm 0,35 b$ & $0,32 \pm 0,17 b$ & $2,11 \pm 0,22 b$ & $S$ & $\mathrm{~S}$ \\
\hline PB 330 & $1,23 \pm 0,38 \mathrm{a}$ & $0,08 \pm 0,01 c$ & $1,67 \pm 0,15 \mathrm{a}$ & $2,21 \pm 0,52 b$ & $0,19 \pm 0,04 c$ & $2,10 \pm 0,21 b$ & $\mathrm{~S}$ & $\mathrm{~S}$ \\
\hline PB 28/59 & $0,76 \pm 0,32 b$ & $0,27 \pm 0,07 b$ & $1,44 \pm 0,13 b$ & $2,15 \pm 0,54 b$ & $0,19 \pm 0,05 c$ & $1,69 \pm 0,18 c$ & $\mathrm{R}_{\mathrm{NP} / \mathrm{A}}$ & $\mathrm{R}_{\mathrm{NP} / \mathrm{A}}$ \\
\hline IRCA 111 & $1,68 \pm 0,41 a$ & $0,19 \pm 0,05 c$ & $1,33 \pm 0,11 b$ & $3,53 \pm 0,58 a$ & $0,19 \pm 0,05 c$ & $1,75 \pm 0,16 c$ & $\mathrm{R}_{\mathrm{T}}$ & $\mathrm{R}_{\mathrm{NP} / \mathrm{A}}$ \\
\hline RRIM 600 & $2,07 \pm 0,66 \mathrm{a}$ & $0,55 \pm 0,14 a$ & $1,58 \pm 0,12 \mathrm{a}$ & $4,32 \pm 0,86 a$ & $0,57 \pm 0,13 a$ & $2,31 \pm 0,19 a$ & $\mathrm{~S}$ & $\mathrm{~S}$ \\
\hline $\mathrm{F}$ & $3,30^{* *}$ & $7,20^{* *}$ & $6,32^{* *}$ & $7,27^{* *}$ & $5,01^{* *}$ & $6,74^{* *}$ & & \\
\hline $\mathrm{P}$ & 0,0008 & $<0,0001$ & $<0,0001$ & $<0,0001$ & $<0,0001$ & $<0,0001$ & & \\
\hline gl & 9 & 9 & 9 & 10 & 10 & 10 & & \\
\hline
\end{tabular}

$\left.{ }^{1,2}\right)$ Médias originais. Análise estatística realizada com os dados transformados em $\log (\mathrm{x}+2)$. Na vertical, médias não seguidas por mesma letra diferem pelo teste de ScottKnott, a $5 \%$ de probabilidade. $\left(^{3}\right)$ Escala de notas: 0, ausência de desfolhamento; 1,0 a 25\%; 2, 25 a 50\%; 3, 50 a 75\%; e 4, acima de 75\%. S: suscetível; $\mathrm{R}_{\mathrm{NP} / \mathrm{A}}$ : resistência por nāo preferência e/ou antibiose; $\mathrm{R}_{\mathrm{r}}$ : resistência por tolerância. 
planta é considerada resistente quando, em determinadas condiçôes, ocorre dano menor do que o dano médio sofrido pelos genótipos cultivados (LARA, 1991). Neste trabalho, o clone RRIM 600 foi considerado o padrão de comparação devido à sua grande importância para a heveicultura paulista. Estima-se que hoje mais de $80 \%$ dos 60 mil hectares com seringueira do Estado estejam plantados com este clone (Gonçalves e Marques, 2008). Apesar de seu bom desempenho com relação à produção e ao vigor (Instituto Agronômico, 2009), é suscetível a ácaros, os quais podem provocar acentuado desfolhamento precoce (Vieira e Gomes, 1999; Vieira et al., 2006; DAud e Feres, 2007). Dessa forma, os estudos na área de resistência a ácaros devem ter por meta identificar materiais mais resistentes do que este clone.

Três tipos de resistência de plantas a pragas podem ser encontrados. Assim, a resistência de uma planta ao desenvolvimento populacional de uma praga pode ser atribuída a um comportamento de não preferência para alimentação, oviposição ou abrigo. Uma segunda possibilidade é a menor população da praga ser resultante de um efeito de antibiose, quando a planta afeta a biologia da praga. Mesmo plantas com grande infestaçáo de uma praga podem ser resistentes por tolerância, caso o ataque resulte em poucos danos (LARA, 1991).

Os clones IAC 15, IAC 40, IAC 300, IAN 3156 e PB 28/59 foram considerados resistentes por náo preferência e/ou antibiose às duas espécies, uma vez que tinham número médio de ácaros menor do que o registrado no clone RRIM 600, nos dois períodos estudados. No presente trabalho, não é possível determinar qual desses dois tipos de resistência está envolvido na resposta observada, ou mesmo se os dois participam do processo. É possível que, em alguns clones, tenha ocorrido um comportamento de fuga dos ácaros, por não encontrarem neles as condiçóes adequadas para a sua permanência, o que seria indicativo de resistência por não preferência. Diferentes estímulos da planta, devido às suas características morfológicas ou químicas podem influenciar a permanência dos ácaros em um hospedeiro (Lara, 1991; Pallini et al., 2001). Por outro lado, em clones resistentes podem ter ocorrido efeito de antibiose pela presença, no substrato alimentar, de substâncias nocivas à espécie, afetando sua biologia. A alimentação de fêmeas nessas condiçóes pode resultar em menor fecundidade e longevidade (VIEIRA et al., 2002).

O clone IRCA 111, com nota média de desfolhamento inferior ao de RRIM 600, nos dois períodos estudados, apesar de níveis semelhantes de infestação de $C$. heveae, foi considerado resistente por tolerância.

Nos clones IAC 35, PB 260 e PB 330, a nota de desfolhamento foi inferior à registrada em RRIM 600, no período 2005/2006, mas semelhante em 2004/2005, ou seja, não houve repetiçáo do comportamento observado (LARA, 1991), deixando dúvidas quanto à presença de fatores de resistência. No presente trabalho, só foram classificados como resistentes os clones com esse comportamento nos dois períodos avaliados; portanto, esses materiais foram considerados semelhantes ao padrão e suscetíveis aos ácaros. Em Itiquira (MT), o clone PB 260 foi mais suscetível a $C$. heveae do que o RRIM 600 (DAuD e Feres, 2007).

No período de 2005/2006 a infestação de $C$. heveae foi maior, ocasionando desfolhamento mais acentuado das plantas (Tabela 2). Na população de $T$. heveae não houve alteraçáo de um período para o outro. Além do maior nível populacional, a infestação de $C$. heveae foi antecipada no segundo período de avaliação. Em 2004/2005, com exceção do clone IAC 300, no qual essa espécie proporcionou pico populacional em março, em todos os outros clones, os maiores níveis do ácaro ocorreram em abril. No segundo período, apenas

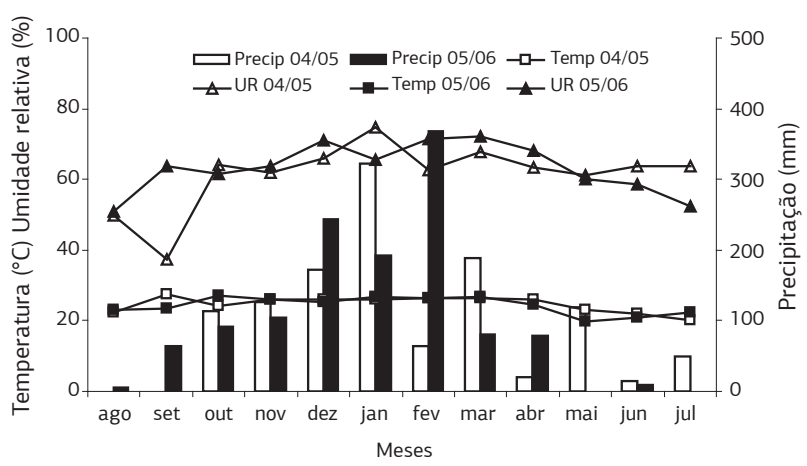

Figura 1. Valores mensais de temperatura média $\left({ }^{\circ} \mathrm{C}\right)$, umidade relativa média (\%) e precipitação pluviométrica total $(\mathrm{mm})$ registrados nos períodos de 2004/2005 e 2005/2006. Votuporanga (SP).

Tabela 2. Médias do número de ácaros por $\mathrm{cm}^{2}$ de $C$. heveae e $T$. heveae, de notas de desfolhamento e de precipitação pluvial mensal, em área de seringueira, nos dois períodos estudados. Votuporanga (SP)

\begin{tabular}{lcccc} 
& Unidade & $\begin{array}{c}\text { Período } \\
\mathbf{2 0 0 4 / 2 0 0 5}\end{array}$ & $\begin{array}{c}\text { Período } \\
\mathbf{2 0 0 5 / 2 0 0 6}\end{array}$ & Teste t \\
\hline C. heveae & Ácaros $\mathrm{cm}^{-2}$ & $1,11 \mathrm{~b}$ & $2,21 \mathrm{a}$ & 5,32 ** \\
\hline T. heveae & Ácaros $\mathrm{cm}^{-2}$ & $0,19 \mathrm{a}$ & $0,21 \mathrm{a}$ & $0,44 \mathrm{~ns}$ \\
Desfolhamento & Notas ${ }^{(1)}$ & $1,50 \mathrm{~b}$ & $1,96 \mathrm{a}$ & 6,32 ** \\
Precipitação $\left({ }^{2}\right)$ & $\mathrm{mm}$ & $114,35 \mathrm{a}$ & $152,80 \mathrm{a}$ & $0,75 \mathrm{~ns}$
\end{tabular}

(')Escala de notas: 0 , ausência de desfolhamento; 1,0 a 25\%; 2,25 a 50\%; 3,50 a 75\%; e 4, acima de 75\%. (2)Média mensal de agosto a fevereiro. ${ }^{* *}$ Teste $t$ de Student significativo a $1 \%$ de probabilidade. 
no clone PB 330 a espécie teve pico populacional em abril. Nos outros materiais ele foi antecipado para janeiro, no caso do clone RRIM 600, fevereiro para IAC 15 e março para os demais. Uma das causas dessa ocorrência pode ter sido a precipitação pluvial no local. Essa espécie é favorecida por ambiente úmido, conforme os dados de Ferla e Moraes (2003), que verificaram, em estudos de laboratório, alta viabilidade da fase de ovo a adulto $(>90 \%)$, com umidade relativa do ar de $90 \pm$ 5\%. Daud e Feres (2007) também observaram maiores populaçóes de $C$. heveae em períodos precedidos por chuvas mais intensas e com maior umidade relativa do ar. Embora não tenha sido detectada diferença significativa entre as médias de precipitação pluvial mensal, de agosto a fevereiro do ano seguinte, nos dois períodos (Tabela 2), houve diferença em relação aos momentos de ocorrência das chuvas (Figura 1). Assim, é possível observar que no período de 2005/2006, as precipitações pluviais em tiveram início em agosto, em contraposiçáo ao período anterior $(2004 / 2005)$ em que as chuvas começaram em outubro. Em 2005/2006, houve também maior intensidade de precipitação pluvial em dezembro. É possível que essa antecipaçáo no período chuvoso possa ter favorecido o desenvolvimento populacional dos ácaros, de forma direta por proporcionar condiçóes climáticas favoráveis ou de forma indireta, interferindo com a condição fisiológica das plantas. A diferença na condição climática pode ser observada pelo valor médio de umidade relativa do ar em setembro de 2004, abaixo de $40 \%$, em comparação com o registrado no ano seguinte, no mesmo mês, 64\% (Figura 1).

Dos clones avaliados, RRIM 600 é o principal clone cultivado no Estado de São Paulo e está incluído na classe I de recomendação de plantio, para utilização em grande escala, em pequenas e grandes propriedades. Por outro lado, os clones IAC 35, IAC 40, IAC 300 e IRCA 111 também fazem parte da lista de recomendação, mas na classe II, para plantio em escala moderada (IAC, 2009). Os outros clones avaliados ainda não são recomendados para plantio.

\section{CONCLUSÃO}

Os clones IAC 15, IAC 40, IAC 300, IAN 3156 e PB 28/59 são resistentes por não preferência e/ou antibiose a $C$. heveae e $T$. heveae. O clone IRCA 111 é tolerante ao ataque de $C$. heveae.

\section{AGRADECIMENTOS}

Os autores agradecem ao Dr. Paulo de Souza Gonçalves e ao Dr. Erivaldo José Scaloppi Junior, da Agência Paulista de Tecnologia dos Agronegócios da Secretaria de Agri- cultura e Abastecimento do Estado de Sáo Paulo, pelo apoio no desenvolvimento desta pesquisa. Aos técnicos José Antonio Agustini e Cristiane Gabas Negrão Souza pela colaboração nas coletas e avaliaçôes laboratoriais. À Coordenadoria de Aperfeiçoamento de Pessoal de Nível Superior - CAPES, pela bolsa de mestrado concedida ao primeiro autor.

\section{REFERÊNCIAS}

DAUD, R.D.; FERES, R.J.F. Dinâmica populacional de ácaros fitófagos (Acari, Eriophyidae, Tenuipalpidae) em seis clones de seringueira no sul do Estado de Mato Grosso. Revista Brasileira de Entomologia, v.51, p.377-381, 2007.

DEMITE, P.R.; FERES, R.J.F. Ocorrência e flutuação populacional de ácaros associados a seringais vizinhos de fragmentos de Cerrado. Neotropical Entomology, v.36, p.117-127, 2007.

FERLA, N.J.; MORAES, G.J. Ácaros (Arachnida, Acari) da seringueira (Hevea brasiliensis Muell. Arg.) no Estado do Mato Grosso, Brasil. Revista Brasileira de Zoologia, v.19, p.867-888, 2002.

FERLA, N.J.; MORAES, G.J. Ciclo biológico de Calacarus heveae Feres, 1992. Revista Brasileira de Entomologia, v.47, p.399-402, 2003.

FERLA, N.J.; MORAES, G.J. Flutuação populacional e sintomas de dano por ácaros (Acari) em seringueira no Estado do Mato Grosso, Brasil. Revista Árvore, v.32, p.365-376, 2008.

FERES, R.J.F.; ROSSA-FERES, D.C.; DAUD, R.D.; SANTOS, R.S. Diversidade de ácaros (Acari, Arachnida) em seringueiras (Hevea brasiliensis Muell. Arg., Euphorbiaceae) na regiâo noroeste do Estado de Sáo Paulo, Brasil. Revista Brasileira de Zoologia, v.19, p.37-144, 2002.

FERREIRA, D.F. Sisvar: um programa para análises e ensino de estatística. Revista Científica Symposium, v.6, p.36-41, 2008.

GONÇALVES, P.S.; MARQUES, J.R.B. Clones de seringueira: influência dos fatores ambientais na produção e recomendação para o plantio. In: ALVARENGA, A.P.; CARMO, C.A.F.S. Seringueira. Viçosa: EPAMIG, 2008. p.179-247.

HERNANDES, F.A.; FERES R.J.F. Review about mites (Acari) of rubbertrees (Heveaspp., Euphorbiaceae) in Brazil. Biota Neotropica, v.6, n.1, 2006a. Disponível em: <http://www.biotaneotropica. org.br/v6n1/pt/abstract?article \pm bn00406012006>. Acesso em: $18 \mathrm{dez} .2009$.

HERNANDES, F.A.; FERES, R.J.F. Diversidade e sazonalidade de ácaros (Acari) em seringal (Hevea brasiliensis, Muell. Arg.) na região noroeste do Estado de São Paulo, Brasil. Neotropical Entomology, v.35, p.523-535, 2006b.

INSTITUTO AGRONÔMICO. Clones: recomendaçôes para plantio. Campinas:IAC, ProgramaSeringueira. 2009. Disponívelem: <http://www.iac.sp.gov.br/UniPesquisa/Seringueira/QuemSomos/ Tecnicas\%20Cultivo/Clones.asp> Acesso em 16 dez. 2009. 
LARA, F.M. Princípios de resistência de plantas a insetos. 2.ed. São Paulo: Ícone, 1991. 336p.

PALLINI. A.; VENZON, M.; JANSEEN, A. Infoquímicos de ácaros mediando interaçôes em teias alimentares. In: VILELA, E.F.; DELLA LUCIA, T.M.C. (Ed.). Feromônios de insetos: biologia, química e aplicaçáo. 2.ed. Ribeirão Preto: Holos, 2001. p.113-120.

RIBEIRO JUNIOR, J.I. Análises estatísticas no SAEG. Viçosa: UFV, 2001. 301p.

VIEIRA, M.R. Pragas e seu controle. In: GONÇALVES, E.C.P. (Coord). A cultura da seringueira para o Estado de São Paulo. 2.ed. Campinas: CATI, 2010. p. 83-101. (Manual Técnico 72)

VIEIRA, M.R.; CAMPOS, A.R.; CASTRO, T.M.M.G.; SILVA, H.A.S.; FIGUEIRA, J.C.; MONTEVERDE，M.S. Resistência de cultivares de algodoeiro ao ácaro branco Polyphagotarsonemus latus (Banks) (Acari: Tarsonemidae). 1. Experimentos em laboratório. Revista Brasileira de Oleaginosas e Fibrosas, v.6, p.545-555, 2002.

VIEIRA, M.R.; GOMES, E.C. Sintomas, desfolhamento e controle de Calacarus heveae Feres, 1992 (Acari: Eriophyidae) em seringueira. Cultura Agronômica, v.8, p.53-71, 1999.

VIEIRA, M.R.; GOMES, E.C.; FIGUEIRA, J.C. Controle químico de Calacarus heveae Feres (Acari: Eriophyidae) em seringueira. Bioassay, v.1, p.9. 2006.

VIEIRA, M.R.; SILVA, H.A.S.; CARDOSO, M.M.; FIGUEIRA, J.C. Progênies de seringueira com potencial para conferir resistência a ácaros (Calacarus heveae Feres e Tenuipalpus heveae Baker). Ciência Rural, v.39, p.1953-1959, 2009.

VIS, R.M.J.; MORAES, G.J.; BELLINI, M.R. Mites (Acari) of rubber trees (Hevea brasiliensis Muell. Arg., Euphorbiaceae) in Piracicaba, state of São Paulo, Brazil. Neotropical Entomology, v.35, p.112-120, 2006. 